There is obviously a considerable role for health promotion activities in this population, especially with reference to smoking and encouraging women to have regular cervical smear tests. However, such health promotion activities must take cognisance of the deprived environment in which the temporarily homeless population lives.

Rates of service utilisation were generally high in the homeless population. General practitioner consultation rates in the 14 days before interview ( $29 \%$ of the sample $(93 / 319)$ ) were almost twice that $(16 \%)$ found in a national sample by the general household survey. ${ }^{5}$ Hospital inpatient utilisation was $12 \%$ per annum as compared with $9 \%$ nationally. ${ }^{5}$ Almost all of the homeless population were registered with general practitioners. However, in many cases the general practitioner was some distance away, probably in their district of origin. In part the high rates of general practitioner registration may reflect the influence of special primary care services for homeless people in Bayswater. There has been considerable debate on whether the high rates of hospital service utilisation by homeless people reflect increased morbidity or lack of access to other services. Our data suggest that, in part, higher morbidity may be a cause of increased service use. However, the high rates of casualty department use may also indicate problems among the homeless population in gaining access to appropriate primary care.

The preliminary results of this survey provide general insight into the nature and characteristics of the temporarily homeless population, which have implications for the planning and provision of health care. Firstly, the population is poor, young, and drawn from minority communities and consists predomi- nantly of parents with preschool age children. Clearly this has implications for the provision of paediatric, obstetric, and family planning services in areas that contain a concentration of this population group. Secondly, service utilisation rates are high in this group and may well reflect these increased rates of chronic morbidity. Additional funding may be merited by those areas that contain concentrations of this population group to take into account their additional health demands. However, if additional funding is made available to districts to provide care for this group, then it is important that such funds should be used not simply to buy additional acute care but to provide appropriate services. Further analysis of this and other surveys will help identify the most appropriate types and styles of services for homeless people.

The survey was sponsored by the North West Thames Regional Health Authority and its constituent districts and undertaken by social and community planning research. Additional funding was provided by Dr L Lessof, of Parkside District Health Authority.

1 Faculty of Public Health Medicine of the Royal College of Physicians. Working group on homelessness and health, housing or homelessness: a public health perspective. London: Faculty of Public Health Medicine, 1991. (Chairperson perspective. Lond

2 Victor CR, Connelly J, Roderick P, Cohen C. Use of hospital services by homeless families in an inner London health district. BMf 1989;299:725-7. 3 Black M, Scheuer MA, Victor CR, Benzeval M, Gill M, Judge K. Homeless and the obligation of acute hospital services in London. London: King's Fund Institute, 1991. (Occasional paper, No 4.)

4 Connelly J, Roderick P, Victor C. Health service planning for the homeless. Public Health 1990;109:13.

Office of Population Censuses and Surveys. General household survey: preliminar results for 1990. London: OPCS, 1991. (OPCS Monitor SS/91/1.)

\title{
Antenatal maternal serum screening for Down's syndrome: results of a demonstration project
}

\author{
Nicholas J Wald, Anne Kennard, James W Densem, Howard S Cuckle, T Chard, L Butler
}

Department of

Environmental and

Preventive Medicine,

Wolfson Institute of

Preventive Medicine,

Medical College of St

Bartholomew's Hospital,

London EC1M 6BQ

Nicholas J Wald, professor

Anne Kennard, antenatal

screening coordinator

James W. Densem, computer manager

Howard S Cuckle, senior

lecturer

\section{Department of}

Reproductive Physiology,

St Bartholomew's Hospital,

London EC1M 6EQ

T Chard, professor

Regional Cytogenetics

Laboratory, Queen

Elizabeth Hospital,

London E2 8PS

L Butler, consultant

cytogeneticist

Correspondence to: Professor Wald.

BMY 1992;305:391-4
Abstract

Objectives-To assess the implementation of antenatal screening for Down's syndrome in practice, using individual risk estimates based on maternal age and the three serum markers: $\alpha$ fetoprotein, unconjugated oestriol, and human chorionic gonadotrophin.

Design-Demonstration project of Down's syndrome screening; women with a risk estimate at term of $\mathbf{1}$ in $\mathbf{2 5 0}$ or greater were classified as "screen positive" and offered diagnostic amniocentesis.

Setting-Hospital and community antenatal clinics in four health districts in London.

Subjects-12 603 women of all ages with singleton pregnancies seen between February 1989 and the end of May 1991, with follow up of the outcome of pregnancy completed to the end of 1991.

Main outcome measures-Uptake of screening, detection rate for Down's syndrome, false positive rate, odds of being affected given a positive result, and uptake of amniocentesis in women with positive screening results, together with the costs of the screening programme.

Results-The uptake of screening was $74 \%$. The detection rate was $48 \%(12 / 25)$, and the false positive rate was $4 \cdot 1 \%$, consistent with results expected from previous work based on observational studies. There was a loss of detection due to the selective use of ultrasound scans among women with positive screening results. One affected pregnancy occurred among 205 reclassified as negative; this illustrated the danger of false negatives occurring in this group and lends weight to the view that if an ultrasound estimate of gestational age is used it should be carried out routinely on all women rather than selectively among those with positive results. The estimated cost of avoiding the birth of a baby with Down's syndrome was about $£ 38000$, substantially less than the lifetime costs of care.

Conclusion-Antenatal maternal serum screening for Down's syndrome is effective in practice and can be readily integrated into routine antenatal care. It is cost effective and performs better than selection for amniocentesis on the basis of maternal age alone.

\section{Introduction}

In 1988, in a study using stored maternal serum samples from pregnancies with and without Down's syndrome fetuses we estimated that antenatal screening for Down's syndrome based on maternal age and the measurement of $\alpha$ fetoprotein, unconjugated oestriol, and human chorionic gonadotrophin in maternal blood would detect $61 \%$ of affected pregnancies with a $5 \%$ false positive rate. This means that about $5 \%$ of women screened would be offered a diagnostic amniocentesis. The estimated detection rate has since been revised from $61 \%$ to $58 \% .^{2}$ In 1989 we set up a screening programme in our local health district, later extending the service to three neighbouring health districts. This 
paper describes the experience of the screening programme until mid-1991, including follow up until the end of 1991, with particular reference to its feasibility and acceptability.

\section{Methods}

Antenatal serum screening for Down's syndrome using the three biochemical tests together with maternal age was introduced in four health districts in London: City and Hackney in 1989, Tower Hamlets and also Newham in 1990, and Waltham Forest in 1991. The populations of these districts are socioeconomically deprived, with a high proportion of ethnic minority residents. The tests were done on the same serum sample used for $\alpha$ feroprotein screening for open neural tube defects, and a special form requesting measurement of $\alpha$ fetoprotein, unconjugated oestriol, and human chorionic gonadotrophin was routinely used in the antenatal clinics. Women were eligible for screening between 15 and 22 weeks of pregnancy. Women who booked too early in pregnancy for the test were asked to return later. The method of screening has been described previously. ${ }^{1}$ Maternal serum $\alpha$ fetoprotein was measured by radioimmunoassay (Amerlex-M $\alpha$ fetoprotein second trimester radioimmunoassay since February 1991 and the North East Thames Radioimmunoassay before), human chorionic gonadotrophin by an immunoradiometric assay (Amerlex-M human chorionic gonadotrophin second trimester radioimmunoassay from June 1990 and the Serono MAIA-Clone Kit before), and unconjugated oestriol by a direct non-extraction radioimmunoassay (Amerlex-M unconjugated oestriol second trimester radioimmunoassay). The concentrations of the three serum markers were expressed in multiples of the median for pregnancies with the same gestational age. The risk of an individual woman having a Down's syndrome term pregnancy was derived as described previously from her age and the trivariate Gaussian frequency distribution of the three serum markers.' The appropriate means and standard deviations of the distributions of $\alpha$ fetoprotein for women who had an ultrasound scan were used and $\alpha$ fetoprotein values adjusted for maternal weight as previously described. ${ }^{3}$

Before the screening programme was implemented, seminars were held to explain the nature of the programme to the nursing and medical staff in the antenatal clinics, an information leaflet was prepared for patients, and a screening coordinator was appointed to administer the screening service and provide individual counselling to patients as needed. Computer assisted test interpretation using the software package "alpha," based on the method of risk estimation previously published,' was used to calculate the risk of having a Down's syndrome term pregnancy for each woman screened. All those with a risk of 1 in 250 or greater were designated "screen positive." The screening test was interpreted in this way for singleton pregnancies only if the gestational age was between 15

Summary of screening results

\begin{tabular}{|c|c|c|c|}
\hline & All ages & $<37$ years & $\geqslant 37$ years \\
\hline No of pregnancies screened & 12603 & 11993 & 610 \\
\hline $\begin{array}{l}\text { No with Down's syndrome } \\
\text { (incidence) }\end{array}$ & $250.0<1000$ & $18(1.5 / 1000)$ & $7(11 / 1000)$ \\
\hline Detection rate & $48 \%(12 / 25)$ & $\begin{array}{l}38 \%(7 / 18) \\
39 \%(1 \cdot 1 / 100)\end{array}$ & $71 \%(5 / 7)$ \\
\hline False-positive ratet: & & & \\
\hline Initially & $5 \cdot 7 \%(718 /(12603-25))$ & $4.9 \%(584 /(11993-18))$ & $22 \%(134 /(610-7))$ \\
\hline $\begin{array}{l}\text { After revised gestation with } \\
\text { ultrasound }\end{array}$ & $4 \cdot 1 \%(514 /(12603-25))$ & $3 \cdot 3 \%(391 /(11993-18))$ & $20 \%(123 /(610-7))$ \\
\hline $\begin{array}{l}\text { Odds of being affected given a } \\
\text { positive result } \\
\text { Uptake of amniocentesis } \ddagger\end{array}$ & $\begin{array}{c}1: 43(12: 514) \\
75 \%(397 /(514+12))\end{array}$ & $\begin{array}{c}1: 56(7: 391) \\
79 \%(316 /(391+7))\end{array}$ & $\begin{array}{c}1: 25(5: 123) \\
63 \%(81 /(123+5))\end{array}$ \\
\hline
\end{tabular}

^ Proportion of Down's syndrome pregnancies with positive screening results.

$\dagger$ Proportion of unaffected pregnancies with positive screening results.

$\ddagger$ Includes 4 cordocenteses and 48 late chorionic villus sampling procedures. and 22 completed weeks of gestation, ideally between 16 and 18 weeks as this is recognised as being the best time for screening for neural tube defects. ${ }^{4}$ Gestational age was determined either from an ultrasound examination at the time of the screening test (performed on $65 \%$ of the women) or from "dates" (time elapsed since the first day of the last menstrual period) if no ultrasound scan was available. In the rare event that neither dates nor scan were available a clinical estimate of gestational age was used. If a screening result was positive and dates had been used to estimate gestational age then an ultrasound scan was performed to check and, if necessary, revise the estimated gestational age. It was recognised that this was likely to lead to a reduction in the proportion of affected pregnancies detected because some of the true positives, as well as false positives, would be reclassified as negative. To minimise this, the policy was changed in August 1989; the revised estimate was used only if the difference between the estimate from dates and the estimate from the scan was 17 days or greater. The choice of 17 days was partly to provide a clear separation between women with differences of two weeks, which were not to be revised, and of three weeks, which were, and partly because the distribution of the differences between the scan and dates estimates was bimodal, with the antimode at 17 days. Antenatal diagnosis using amniocentesis was offered to all women designated as screen positive once an ultrasound scan had been performed. A termination of pregnancy was offered to women who had a karyotypic diagnosis of Down's syndrome.

The uptake of screening was taken as the number of women who were screened divided by the number of deliveries. The number of deliveries was shown to be a reasonable estimate of the number of women who booked for antenatal care; in a six month sample at one hospital there were 2025 deliveries and 1921 women who booked. Figures on antenatal booking were not available for the whole period. Women with twin pregnancies were excluded from the analysis. Pregnancies associated with Down's syndrome and infants with Down's syndrome were identified using the records of the cytogenetic laboratory at Queen Elizabeth Hospital, which was responsible for performing all karyotyping in the four districts. Records were examined up to 31 December 1991 to ensure complete ascertainment.

\section{Results}

A total of 12603 women with singleton pregnancies were screened between February 1989 and May 1991. The uptake of screening was $74 \%$. The table summarises the screening results for women of all ages and separately for those under 37 years and those 37 or more. The overall detection rate among screened women was $48 \%(12 / 25,95 \%$ confidence interval $28 \%$ to $69 \%$ ) and the false positive rate was $4 \cdot 1 \%$ $(514 / 11578 ; 3 \cdot 7 \%$ to $4 \cdot 4 \%)$. The odds of being affected, given a positive screening result, were 1 in 43, and 397 ( $75 \%$ ) of the 526 women with positive results accepted the offer of amniocentesis. The median gestational age at the time of screening was 17 weeks 0 days; $90 \%$ were screened by 19 weeks 1 day.

Ultrasound scanning was widely used. Women who had not already had an ultrasound examination and had a positive screening result then had a scan to revise the estimate of gestation. Thirteen of the affected pregnancies were initially classified as positive, and one was reclassified as negative after gestational age was revised on the basis of an ultrasound scan examination; 204 out of 718 unaffected pregnancies with positive screening results were similarly reclassified as screen negative. The observed risk of Down's syndrome in the pregnancies with positive results that were sub- 
sequently reclassified as negative was therefore 1 in 205 (greater than the estimated cut off risk of 1 in 250 based on unselected screened women), though with only one case of Down's syndrome it would require considerably more data to obtain a reliable estimate of the risk in this group.

Of the 12 screen positive women who were subsequently found to have an affected pregnancy, one declined amniocentesis. Nine of the remaining 11 had a termination of pregnancy and one declined; one travelled overseas and could not be traced.

Among screen positive women, younger women were more likely to accept the offer of amniocentesis than older women, a difference that was independent of their risk of having a fetus with Down's syndrome; the acceptance rate was $79 \%(316 / 398)$ among women aged less than 37 years, but only $63 \%(81 / 128)$ among older women $(p<0 \cdot 01)$. The level of risk itself had a small influence on the decision to have amniocentesis; when the risk was greater than about 1 in 50 there was a greater tendency to accept amniocentesis, an effect that was more apparent among the older women than the younger women. Among women aged 37 years or over, $77 \%(20 / 26)$ accepted amniocentesis if the risk was 1 in 50 or greater compared to $60 \%(61 / 102)$ of those with a lower risk. The uptake of amniocentesis also varied according to the ethnic origin of the woman. Among white women it was $84 \%$, Afro-Caribbean $78 \%$, Pakistani $75 \%$, Indian $74 \%$, Bangladeshi $42 \%$, and others $79 \%$.

\section{Discussion}

Our demonstration project shows that maternal serum screening for Down's syndrome can be carried out effectively on a community basis as a routine part of antenatal care. The uptake of screening was $74 \%$ and the uptake of amniocentesis in screen positive women was $75 \%$; before serum screening with the policy of offering amniocentesis to women of advanced maternal age the uptake was under $50 \%$.

The detection rate was $48 \%(12 / 25)$ and the false positive rate was $4 \cdot 1 \%$. A similar demonstration project performed in the United States yielded a detection rate of $58 \%(21 / 36)$ and a false positive rate of $3.8 \% .^{6}$ These results are compatible with the figures predicted in our recent publication specifying revised screening parameters. ${ }^{2}$ When gestation is based on "dates" we predicted a detection rate of $58 \%$ and false positive rate of $5 \cdot 2 \%$; and when based on ultrasound, rates of $65 \%$ and $4 \cdot 6 \%$. A detailed comparison of observed and expected results is complicated by the change to using log rather than absolute unconjugated oestriol values and by the non-systematic use of ultrasonography in ordinary practice. The wide confidence limits on the observed detection rate $28 \%$ to $69 \%$ ) mean that the predicted results are a better guide to expected performance than the observed results in this dataset would indicate.

Using ultrasound selectively to revise gestational age in women with positive results on screening will reduce the numbers of both true positives and false positives. One affected pregnancy was missed in spite of the " 17 day rule," and two more would have been missed if the 17 day rule had not been introduced. Such revision of the test result in women who had initially been told they were screen positive is particularly distressing. The remedy is to perform a scan on all women at the time of screening, thereby avoiding the need to revise the estimate of gestational age after a woman has been screened and found to be positive.

All Down's syndrome pregnancies in the women who were screened were likely to have been ascertained because one laboratory is responsible for all the karyotype examinations in the districts that took part in the study. Also, the number of Down's syndrome pregnancies $(25)$ is actually slightly greater than the number expected from the age distribution of the population screened and the prevalence of Down's syndrome in births to women of specific ages, after allowing for the spontaneous fetal loss of Down's syndrome (23).

The screening programme showed the acceptability of this type of screening. There was no difficulty in abandoning screening based on maternal age alone (or on maternal age and serum $\alpha$ fetoprotein) in favour of screening using all four markers. It was clear from discussions with the screened women during counselling that those who were older than average appreciated having the additional information about the risk of an affected pregnancy, and many were pleased to avoid amniocentesis if their risk was low. Only $9 \%$ of women aged 37 years or more with a risk estimate of less than 1 in 250 (that is, screen negative) chose to have amniocentesis, and none of these women had an affected pregnancy.

The demonstration project provided information on the costs of screening. To screen 20000 women the extra laboratory costs in connection with the measurement of unconjugated oestriol and human chorionic gonadotrophin, together with those costs needed for interpretation, counselling, education, and administration on a district basis, would amount to about $£ 290000$ ( $\alpha$ fetoprotein is already measured in screening for neural tube defects). The cost of 1000 (approximately $5 \%$ ) amniocenteses and karyotypes, estimated at $£ 150$ each, would be $£ 150000$. In 20000 women the expected number of cases of Down's syndrome at term would be 26 , of which 16 would be detected by serum screening. If all women with positive screening tests accept amniocentesis and all women with an affected fetus accept a termination of pregnancy (at $£ 1000$ each), the cost for each Down's syndrome birth avoided would be about $£ 28500$. In the demonstration project $75 \%(9 / 12)$ accepted the offer of amniocentesis and there was a $90 \%(9 / 10)$ acceptance of termination, making the cost for each Down's syndrome birth avoided $£ 38000$. This is somewhat higher than the estimate of Sheldon and Simpson ( $\$ 29000)$, which was based on somewhat different underlying costs and acceptance values. ${ }^{8}$ The cost of about $£ 38000$ is substantially less than the lifetime costs of care, estimated in 1987 at about $£ 120000$ (after appropriate discounting). ${ }^{9}$ The most important reason for screening, however, is not financial: it is the avoidance of handicap and of distress to the families concerned.

The demonstration project has shown that antenatal serum screening for Down's syndrome is effective and acceptable in practice. It was readily integrated into antenatal care and was shown to be cost effective. The NHS should ensure that antenatal maternal serum screening for Down's syndrome is available throughout Britain.

We thank Rossana Stone, Lynne George, Les Perry, Muriel Kitau, and Derek Hartey for performing the serum assays; Joan Sneddon for helping to set up the screening programme; Susan Player for assisting with the administration of the programme; David Smith for computing assistance; Tom Spencer in the cytogenetic laboratory; Malcolm Law for his comments; and the John Ellicott Centre for assisting with the linkage of maternity data. We thank the antenatal clinic staff at the Homerton Hospital, the Royal London Hospital, Newham Hospital, and Whipps Cross Hospital and the following consultants who helped introduce the programme in their own districts: $\mathrm{Mr} \mathrm{H}$ Annan, $\mathrm{Mr} \mathrm{R}$ Baldwin, $\mathrm{Mr} \mathrm{T}$ Beedham, Miss J M Boulton, Mr O Djahanbakhch, Dr P Foley, Mr M Gillard, Mr D Glyn Evans, Professor J D Grudzinskas, Mr J C Hartgill, Mr C Hudson, Mr D L MacMillan, Mr A A Naftalin, Mr D Oram, Mr V Pathasundaram, Miss A P Roberts, Mrs W D Savage, Mr M E Setchell, Mr D Viniker, and Mr N Wathen. 
We thank the Harris Trust for providing the initial support for this demonstration project and the North East Thames Regional Health Authority and Amerlite Diagnostics Limited for additional grants which together funded the whole programme.

1 Wald NJ, Cuckle HS, Densem JW, Nanchahal K, Royston P, Chard T, et al. Maternal serum screening for Down's syndrome in early pregnancy. $B M$ $1988 ; 297: 883-7$

2 Wald NJ, Cuckle HS, Densem JW, Kennard A, Smith D. Maternal serum screening for Down's syndrome: the effect of routine ultrasound scan determination of gestational age and adjustment for maternal weight. $\mathrm{Br} f$ Obstet Gynaecol 1992;99:144-9.

3 Cuckle HS, Wald NJ, Thompson SG. Estimating a woman's risk of having a pregnancy associated with Down's syndrome using her age and serum alphafetoprotein level. Br f Obstet Gynaecol 1987;94:387-402.

4 Wald NJ, Cuckle H, Brock DJH, Peto R, Polani PE, Woodford FP. Maternal serum alpha-fetoprotein measurement in antenatal screening for anencephaly and spina bifida in early pregnancy. Report of the UK collaborative study on alpha-fetoprotein in relation to neural-tube defects. Lancet, $1977 \cdot ; \cdot 1323-32$. 5 Cuckle H, Nanchahal K, Wald N. Birth prevalence of Down's syndrome in England and Wales. Prenatal Diagnosis 1991;11:29-34.

6 Haddow JE, Palomaki GE, Knight GJ, Williams J, Pulkkinen A, Canick JA, et al. Combining maternal serum alpha-fetoprotein, unconjugated estriol and t al. Combining maternal serum alpha-fetoprotein, unconjugated estriol and human chorionic gonadotropin measurements with maternal age to screen fetal Down syndrome: A demonstration project. N Engl $f$ Med (in press).

Wald NJ, Cuckle HS. Biochemical screening. In: Brock D, Rodeck C, Ferguson-Smith MA, eds. Prenatal diagnosis and screening. London: Churchill Livingstone (in press).

8 Sheldon TA, Simpson J. Appraisal of a new scheme for prenatal screening for Down's syndrome. BMY 1991;302:1133-6.

9 Gill M, Murday V, Slack J. An economic appraisal of screening for Down's syndrome in pregnancy using maternal age and serum alpha fetoprotein concentration. Soc Sci Med 1987;24:725-31.

(Accepted 19 June 1992)

\title{
Laparoscopic cholecystectomy as a safe and effective treatment for severe acute cholecystitis
}

\author{
R G Wilson, I M C Macintyre, S J Nixon, J H Saunders, J S Varma, P M King
}

\section{Abstract}

Objective-To evaluate the feasibility and safety of laparoscopic cholecystectomy in severe acute cholecystitis.

Design-Analysis of data collected prospectively from a consecutive series of $\mathbf{3 5 0}$ laparoscopic operations.

Setting-Two general surgical units in a teaching hospital.

Subjects -31 patients with a diagnosis of severe acute cholecystitis based on clinical examination, investigation results, and operative findings.

Interventions - Initial intravenous fluids and broad spectrum antibiotics followed by laparoscopic cholecystectomy within $\mathbf{7 2}$ hours of presentation.

Main outcome measures-Failure to complete the operation laparoscopically, length of postoperative stay in hospital, early postoperative morbidity, interval from operation to full activity, and return to work.

Results-Laparoscopic cholecystectomy was attempted in 19 patients with empyema of the gall bladder and 12 who had severe cholecystitis which failed to settle on medical management. A total of 29 operations were successfully completed with two conversions to open surgery. Two minor postoperative complications occurred, and one case of retained common bile duct stones with jaundice was treated by endoscopic retrograde cholangiopancreatography and papillotomy. Median postoperative hospital stay was two days, with return to normal activity in seven days and to work in two weeks. There were no deaths related to the operation.

Conclusions-In the presence of severe acute cholecystitis laparoscopic cholecystectomy is feasible in most patients, with minimal risk of injury to surrounding structures and considerable benefits.

Department of Surgery, Western General Hospital, Edinburgh EH4 2XU R G Wilson, surgical registrar I M C Macintyre, consultant surgeon

S J Nixon, consultant surgeon J H Saunders, consultant surgeon

J S Varma, senior registrar

P M King, senior registrar

Correspondence to:

Mr Wilson.

BMF 1992;305:394-6 with a varied degree of technical success in limited series. ${ }^{3-5}$ Since the introduction of laparoscopic surgery in our hospital we have attempted laparoscopic cholecystectomy in all patients presenting with symptomatic gall stones, even when acute inflammation is suspected. We describe our initial experience in those patients who presented with empyema or severe acute inflammation of the gall bladder.

\section{Patients and methods}

Since May 1990 laparoscopic cholecystectomy has been our standard approach to surgery for symptomatic gall stone disease. Up to October 1991, 350 consecutive operations had been attempted with only seven failures. Prospective documentation was kept on all patients regarding presentation, investigations, operation, complications, and early follow up. Eighty six patients with acute pain had been admitted to this hospital as emergencies. Forty four settled on conservative treatment, were discharged, and were readmitted for interval cholecystectomy at a later date. Forty two patients were operated on during their first admission, 31 of whom had definite clinical and laparoscopic evidence of severe acute cholecystitis. This subgroup of 31 formed the basis of this study (figure). The

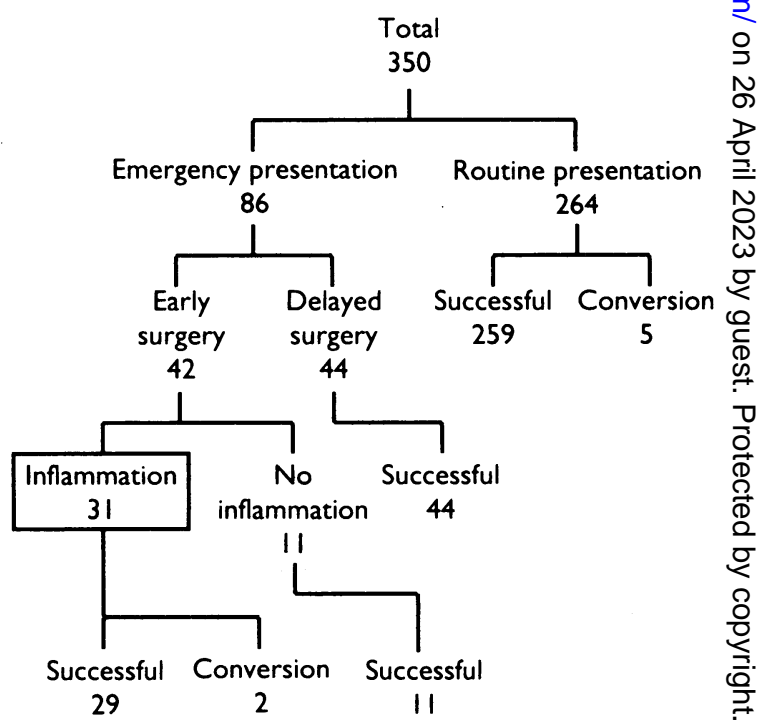

Details of 350 consecutive laparoscopic cholecystectomies on patients presenting with symptomatic gall stone disease, indicating the 31 patients who had definite clinical and laparoscopic evidence of severe acute cholecystitis 